\title{
Prognostic implications of node metastatic features in OSCC: A retrospective study on 121 neck dissections
}

\author{
MARIA CONTALDO $^{1 *}$, ANTONIO DI NAPOLI ${ }^{1 *}$, GIUSEPPE PANNONE ${ }^{2}$, RENATO FRANCO $^{3}$, FRANCO IONNA $^{4}$, \\ ANTONIA FEOLA $^{5}$, ALFREDO DE ROSA ${ }^{1}$, ANGELA SANTORO ${ }^{2}$, CAROLINA SBORDONE $^{6}$, \\ FRANCESCO LONGO $^{4}$, DANIELA PASQUALI $^{7}$, CARLA LORETO $^{8}$, FILIPPO RICCIARDIELLO $^{9}$, \\ GENNARO ESPOSITO $^{10}$, LUIGI D'ANGELO ${ }^{1}$, ANGELO ITRO ${ }^{1}$, PANTALEO BUFO $^{2}$, \\ VINCENZO TOMBOLINI $^{11}$, ROSARIO SERPICO ${ }^{1}$ and MARINA DI DOMENICO ${ }^{5,12}$
}

\begin{abstract}
${ }^{1}$ Multidisciplinary Department of Medical-Surgical and Odontostomatological Specialties, Second University of Naples, I-80138 Naples; ${ }^{2}$ Department of Clinical and Experimental Medicine, University of Foggia, I-71122 Foggia; Sections of ${ }^{3}$ Pathological Anatomy, and ${ }^{4}$ Head and Neck Surgery, National Cancer Institute-G. Pascale Foundation, I-80131 Naples; ${ }^{5}$ Department of Biochemistry, Biophysics and General Pathology, Second University of Naples, I-80131 Naples;

${ }^{6}$ Maxillo-Facial Surgery Chair of Maxillo-Facial Surgery, School of Medicine, University of Naples, I-80131 Naples;

${ }^{7}$ Department of Endocrinology, Second University of Naples, I-80131 Naples; ${ }^{8}$ Department of Bio-Medical Sciences, Section of Anatomy, University of Catania, I-95123 Catania; ${ }^{9}$ Section of Otolaryngology, University of Naples Federico II, I-80138 Naples; ${ }^{10}$ Section of II Orthopaedic Cardarelli Hospital, I-80131 Naples; ${ }^{11}$ Department of Radiology, Oncology and Pathological Anatomy Sciences, Sapienza University of Rome, I-00161 Rome, Italy; ${ }^{12}$ Sbarro Institute for Cancer Research and Molecular Medicine, Center of Biotechnology, Temple University, Philadelphia, PA 19122, USA
\end{abstract}

Received May 1, 2013; Accepted June 28, 2013

DOI: $10.3892 /$ or.2013.2779

\begin{abstract}
Lymph node metastases are responsible for shorter survival in oral squamous cell carcinoma (OSCC). The aim of the present study was to assess the node metastasis frequency and survival according to the node metastasis features in 121 neck dissections (NDs) performed for OSCC, identifying evidence-based correlations and contrasts with previous literature. The retrospective study involved 121 patients affected by OSCC who had undergone modified radical ND (MRND) for therapeutic, elective reasons or after intraoperative positivity to metastasis of sentinel lymph nodes (SLN+). Node metastasis frequency and behaviour (typical vs. atypical)
\end{abstract}

Correspondence to: Dr Marina Di Domenico, Department of Biochemistry, Biophysics and General Pathology, Second University of Naples, Via Luigi De Crecchio 7, I-80138 Naples, Italy

E-mail: marina.didomenico@unina2.it

Dr Alfredo De Rosa, Multidisciplinary Department of MedicalSurgical and Odontostomatological Specialties, Second University of Naples, Via Luigi de Crecchio 6, I-80138, Naples, Italy

E-mail: alfredo.derosa@unina2.it

*Contributed equally

Key words: oral cancer, lymph node metastasis, micrometastasis, extracapsular spread, survival, neck dissection, sentinel lymph node, skip metastasis and their number and distribution according to pre-surgical cTNM cancer staging were considered and overall survival Kaplan-Meier curves were calculated for each group in order to compare mortality according to ND type (elective, therapeutic, after SLN+), lymph node metastatic pattern (typical or atypical), size (micrometastasis vs. macrometastasis) and number. Results showed statistically significant different overall survival according to pre-surgical staging, number of lymph nodes harvested and intent to surgery. Sentinel lymph node resulted in the sole positive node affected by metastasis in small cT1- cT2/cN0 OSCC and an ND subsequent to its positivity during intraoperative assessment may be considered an overtreatment.

\section{Introduction}

Oral squamous cell carcinoma (OSCC) is the sixth most common cancer internationally, accounting for $\sim 5 \%$ of all malignant tumours worldwide (1). OSCCs commonly metastasize to cervical lymph nodes. Each tumour generally metastasizes in a particular group of cervical lymph nodes and the principles and criteria governing neck dissection (ND) are based on the primary tumour characteristics, according to the cTNM staging and its primary site $(2,3)$.

Cervical lymph node metastases (LNMs) are key malignancy criteria in OSCC (4). Their presence influences the therapeutic plan and prognosis, since it is associated with a $50 \%$ decrease in survival $(5,6)$.

The selection of OSCC cases requiring ND depends mainly on the clinical TNM staging (cTNM). cTNM provides a stage 
grouping based on the extent of the primary tumour ( $\mathrm{T}$ score), the involvement of the regional, cervical, lymph nodes $(\mathrm{N}$ score) and the detection of distant metastases (M score) $(2,3,7)$. These parameters are accurately quantified after performing a series of clinical-instrumental examinations such as PET, total body CT scan, neck echo-color Doppler, fibrolaryngoscopy, esophageal-gastric-duodenoscopy, bronchoscopy and, if any doubts persist, fine needle aspiration biopsy (FNAB) and biopsy must be also performed.

Once clinical T, $\mathrm{N}$ and $\mathrm{M}$ parameters have been defined, a ND is mandatory for the OSCCs showing cervical LNMs (any $\mathrm{cT} / \mathrm{N}+$ ) and for locally advanced primary tumours (cT3 or cT4) with clinically undetectable LNMs (cN0).

Based on the intent or purpose, NDs have been also classified into therapeutic and elective. Therapeutic NDs are performed in OSCCs with cervical metastases detected in clinical preoperative setting (any $\mathrm{T} / \mathrm{N}+$ ). Elective NDs are selected for locally advanced primary tumours (T3 or T4) with clinically undetectable LNMs (cN0) (8). NDs are also performed in the cases of small primary tumours clinically negative to node involvement (cT1-T2/N0) that during intraoperative assessment of their sentinel lymph node reveal positivity to metastasis (9).

Both ND anatomical extent and involvement of surrounding structures are related to the node levels involved in the dissection and they are planned on the basis of the OSCC primary site; correlations between primary site of the cancer and level of metastasis have been demonstrated and, to date, they aid the surgeon in the surgical ND management (10).

Currently, NDs are classified into four basic procedures according to the extent of different cervical lymph node groups and surrounding structures: radical ND, modified radical ND, (MRND) extended ND and selective ND $(8,11)$.

Following histopathological assessment of the tumour margins of excision and after the evaluation of the involvement of the surrounding structures, adjuvant therapy may also be performed: radiotherapy for T4 tumours with free surgical margins and/or $\geq \mathrm{N} 2$; and both radiotherapy and chemotherapy for tumours with any $\mathrm{N}+$ plus extracapsular spread (ECS) and ones with any $\mathrm{T}$ and positive or close margins or perineural invasion and/or neoplastic vascular embolization. T1-3 tumours with free margins and $\mathrm{pN} 0 / \mathrm{pN} 1$ do not require adjuvant treatment $(12,13)$.

Despite the progress in pre-surgical instrumental examinations (head and neck CT scan, neck echo-color Doppler, fibrolaryngoscopy, esophageal-gastric-duodenoscopy, bronchoscopy and PET), clinical lymph node staging is not completely error-free due to false positivity in the presence of reactive lymph nodes, non metastatic lymph node enlargement and false negativity for small- or micrometastases clinically undetectable $(14,15)$. Finally, the controversial role of sentinel lymph node positivity and the surgical morbidity after ND have led to the evaluation of alternative and super-selective surgeries in order to reduce the overtreatments (15,16-18).

For these reasons, further in-depth studies regarding the behaviour of lymph node cervical metastases may be useful to refine therapeutic management, thereby decreasing the overtreatment-related morbidity and mortality.

The aim of the present study was to define LNM frequency, topographic distribution, size (micrometastases vs. macrome- tastases) and histological pattern correlating them with the clinical features of primary tumour in 121 OSCC patients who had undergone ND, considering the survivals related to their presence/absence and the morbidity related to the negative ND and due to the neck surgery, comparing our results with the literature and suggesting an evidence-based re-evaluation of the therapeutic approach to ND.

\section{Materials and methods}

Study population and clinical pathological data. Resection specimens from 121 patients who had undergone ND surgery for OSCC at the National Cancer Institute of Naples, 'G. Pascale', Italy, between the years 1993-2004, formed the basis of the present retrospective analysis. All patients underwent MRND for OSCC $(11,19)$. Patients who had had previous surgery (other than diagnostic biopsy) were excluded. Throughout the time period of the study, the resection specimens were performed by the same surgical team and were assessed by the same pathological team. Pathological report and topography of the extent and location of the metastatic disease for each patient were reviewed and number, size and histological patterns of LNM-positive cases $(\mathrm{pN}+)$ were re-evaluated according to previous literature $(14,15,20,21)$ in order to calculate frequency, distribution and other significant statistical correlations existing between primary tumour features and $\mathrm{pN}+$.

The series comprised 80 males (mean age of $63.10 \pm 10.79$ years; range, 30-83 years, median 63 years) and 41 females (mean age, $63.12 \pm 15.19$ years; range, 25-86 years, median, 65 years). The cTNM staging was assessed according to the 6th edition AJCC (2) since data refer to the period between 1999-2004. Patient demographic and clinical characteristics of the 121 cases are summarized in Table I.

Pre-operative, operative and post-operative protocols. At the National Cancer Institute of Naples 'G. Pascale', Italy, the selection of OSCC cases requiring ND depends on the cTNM (2). cTNM parameters are accurately quantified after performing a series of clinical-instrumental examinations such as PET, total body CT scan, neck echo-color Doppler, fibrolaryngoscopy, esophageal-gastric-duodenoscopy, bronchoscopy and, if any doubts persist, fine needle aspiration biopsy (FNAB) and biopsy.

According to the literature, MRNDs are considered mandatory for the OSCCs clinically showing cervical LNMs (any cT/N+ and therapeutic ND), for locally advanced primary tumours with clinically undetectable LNMS (cT3/N0 and cT4/ $\mathrm{cN} 0$, elective ND) and in the cases of small primary tumours clinically negative to node involvement (cT1-T2/N0) that during intraoperative assessment of their sentinel lymph node reveal a positivity to metastasis $(\mathrm{SLN}+)(8,9)$.

For the ND surgical approach, we adopted MRND (10), thus involving levels I-IV for all oral sub-sites, except for trigone, posterior tongue and anterior pillar where ND extended until level V; level IIB was always comprised.

After surgery and histopathological assessment of the margins of excision of the primary tumour and after the evaluation of the involvement of the surroundings structures, adjuvant therapy was performed; radiotherapy for T4 tumours 
Table I. Patient demographics and clinical characteristics.

\begin{tabular}{lc}
\hline & $\mathrm{N}(\%)$ \\
\hline Gender (male/female) & $80 / 41(66 / 34)$ \\
Male mean age, years (range) & $63.10(30-83)$ \\
Female mean age, years (range) & $63.12(25-86)$ \\
Primary T site & \\
Tongue & $53(44)$ \\
Floor of the mouth & $23(19)$ \\
Cheek & $4(3)$ \\
Trigone & $17(14)$ \\
Oropharynx & $8(7)$ \\
Palate & $3(2)$ \\
Fornix & $10(9)$ \\
Not specified & $3(2)$ \\
Histologic grade & \\
Low & $35(29)$ \\
Intermediate & $65(54)$ \\
High & $21(17)$ \\
AJCC stage & \\
I & \\
II & $11(9)$ \\
III & $30(25)$ \\
IV & $28(23)$ \\
\hline
\end{tabular}

with free-surgical margins and/or $\geq \mathrm{N} 2$, and both radiotherapy and chemotherapy both for tumours with any $\mathrm{N}+$ plus ECS, tumours with any $\mathrm{T}$ and positive or close margins or perineural invasion and/or neoplastic vascular embolization tumours T1-3 with free margins and $\mathrm{pN} 0 / \mathrm{pN} 1$ were not enrolled for adjuvant treatments $(12,13)$.

Since data refer to patients recruited from 1999 to 2004 , prior to the last Cancer Staging Atlas publication, we considered the previous edition (2); hence, T4 tumours were not distinguished in T4a and T4b, consequently, stage IV has not been subclassified into stages IVa, IVb and IVc (7).

Histopathological node metastasis classification. All 121 lymph node specimens dissected were histopathologically re-evaluated for the present study. In order to detect micrometastases and to confirm the involvement of lymph nodes, the standardized sectioning protocol was performed. One H\&E stained section was prepared from each block and examined for the presence of nodal involvement by tumour. If present, metastatic disease was reported. If node was negative or equivocal for metastatic disease, or positive for micrometastases, the removed lymph nodes were also serially sectioned at $25-\mu \mathrm{m}$ intervals $4 \mu \mathrm{m}$ thick and alternately stained with $\mathrm{H} \& \mathrm{E}$ and immunohistochemical staining (IHC) using anticytokeratin and EMA antibodies (Fig. 1) as described below. This pattern was continued throughout the entire block. Each immunostained component was always compared with adjacent sections stained by H\&E.

Woolgar's classification criteria were considered in order to classify the type of metastasis $(21,22)$. Woolgar distin-
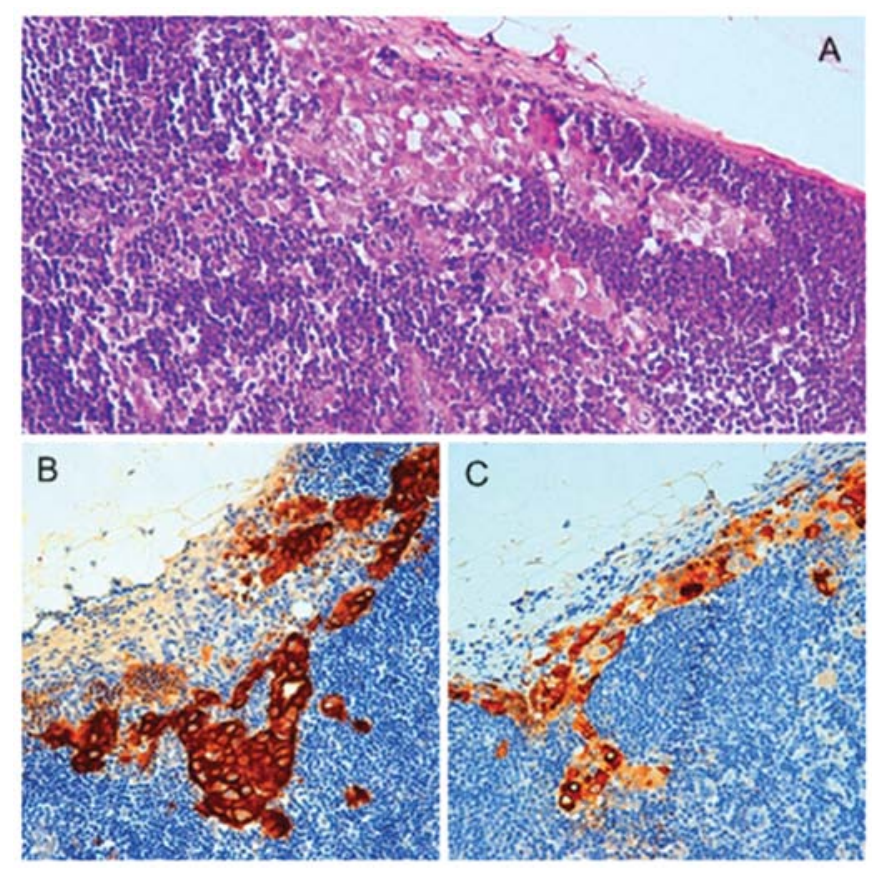

Figure 1. Immunohistochemical H\&E staining of a node metastasis showing cytokeratin and EMA positivity confirming the presence of OSCC metastases in the lymph nodes evaluated.

guished metastatic lymph nodes into two groups with different prognosis and features: typical metastatic pattern as 'orderly involvement of successive anatomical nodal levels, creating an inverted cone with maximum volume and maximum ECS at levels I or II and a gradual reduction in the volume/extent of metastasis at the numerically higher levels' (21), vs. atypical metastatic pattern, termed 'aberrant' by Woolgar, and characterized by various features. The ones referring to the atypical pattern and considered in the present study were the involvement of 'other' anatomical groups of nodes (including parapharyngeal, facial, buccal, lingual and sublingual nodes), involvement of controlateral cervical lymph nodes, skipping of anatomical levels other than level I and the presence of a single micrometastasis (21).

Once defined, the histopathological features of node metastases were correlated with the clinical and histopathological features of the primary tumour in order to establish statistical and prognostic correlations.

Immunohistochemistry. Histological and immunohistochemical analyses were performed on formalin-fixed, paraffin-embedded tissue samples. Immunostaining was performed using the linked streptavidin-biotin horseradish peroxidase technique (LSAB-HRP). Antigen retrieval was performed by microwave heating, a first time for $3 \mathrm{~min}$ at $650 \mathrm{~W}$, a second and a third time for $3 \mathrm{~min}$ at $350 \mathrm{~W}$, the slides immersed in $10 \mathrm{mM}$ citrate buffer $\mathrm{pH}$ 6.0. After heating, the sections were blocked for $60 \mathrm{~min}$ with $1.5 \%$ horse serum (Santa Cruz Biotechnology) diluted in PBS buffer before reaction with the primary antibody $(\mathrm{Ab})$. The primary monoclonal antibodies anti CK AE1/AE3 (dilution 1:50, $\mathrm{pH} 6.0$; Dako, Carpinteria, CA, USA) and EMA (dilution 1:75, with protein K; Dako) were incubated overnight. After two washes 
with PBS, the slides were treated with biotinylated speciesspecific secondary antibodies and streptavidin-biotin enzyme reagent (Dako, Glostrup, Denmark), and the colour developed by 3,3'-diaminobenzidine tetrahydrochloride. Sections were counterstained with Mayer's hematoxylin and mounted using xylene-based mounting medium. In negative controls, the primary antibody was omitted. The results of the IHC were separately evaluated by two independent observers by carefully examining the entire section with an optical microscope (Olympus BX41). For each case, the presence and the extent of positive cells in all sections examined was determined. Isolated tumour cells (ITCs) are defined as tumour cell clusters that are not $>0.2 \mathrm{~mm}$ in largest diameter and are denoted as lymph node negative (pNO[i+]). Micrometastases are defined as metastases that are $>0.2 \mathrm{~mm}$ in diameter but $\leq 2 \mathrm{~mm}$, denoted as lymph node positive (pN1mi). Carcinoma macrometastases measured $>2 \mathrm{~mm}$ in maximum extent. Two investigators experienced in oral pathology blindly and independently examined the study sections initially, and then they evaluated together the histopathological and immunostained sections until they reached an agreement.

Statistical analysis. Data were analysed by the GraphPad Prism software version 5.0 for Windows (GraphPad Software, San Diego, CA, USA; www.graphpad.com) and Excel Microsoft Office. Differences among the groups were estimated using the one-way analysis of variance (ANOVA) and the Student-Newman-Keuls test. Only P-values <0.05 were considered significant. Overall survivals in the different groups were calculated by the Kaplan-Meier curves and logrank (Mantel-Cox) test was applied for comparing survival probabilities. The pathological positivity of the ND $(\mathrm{pN}+)$ and the type of LNMs were correlated with the site of primary tumour. The percentages of positive node metastases $(\mathrm{pN}+)$ in elective, therapeutic and secondary to SLN+ NDs and the number of lymph nodes harvested were also evaluated.

\section{Results}

Differentiation degree, cTNM staging and pN status of 121 cases. Patient demographics and characteristics such as gender, OSCC site, primary tumour differentiation grading and cTNM staging according to the international guidelines (2) of the 121 OSCCs considered are shown in Table I.

At pre-surgical clinical-instrumental evaluation, 61/121 cases (50.4\%) were considered positive (cN+) and 60/121 (49.6\%) negative (cN0) to node metastases. The histopathological lymph node assessment subsequent to ND revealed at least one node metastasis in 56/121 NDs, thus considered pathologically positive lymph nodes $(\mathrm{pN}+)$ and no node metastasis in the remaining 65 cases, which were considered pathologically negative lymph nodes (pN0). The percentages of true positive $(\mathrm{cN}+$ and $\mathrm{pN}+)$, true negative ( $\mathrm{cNO}$ and $\mathrm{pN} 0)$, false positive $(\mathrm{cN}+$ and $\mathrm{pN} 0)$ and false negative $(\mathrm{cN} 0$ and $\mathrm{pN}+)$ were 87, 95, 13 and 5\% respectively, as reported with details of accuracy, sensitivity, specificity, and positive and negative predictive values in Fig. 2.

The $\mathrm{pN}+$ distribution according to the grading, staging MRND intent and, conversely, the $\mathrm{pN}+$ frequency in each group are reported in Table II.

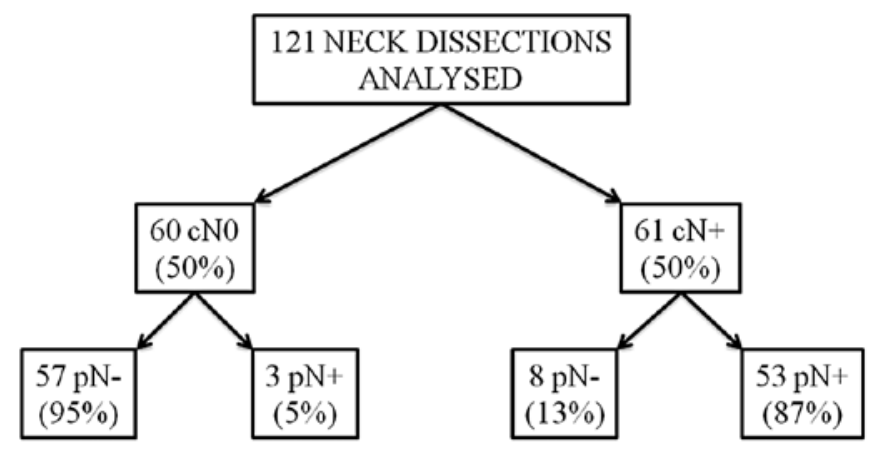

Figure 2. Flow chart showing the cases analysed in the study and the accuracy, sensitivity, specificity and predictive values of the clinical instrumental approach. Accuracy, 0.909; sensitivity, 0.946; specificity, 0.877; PPV, positive predictive value, 0.869 . NPV, negative predictive value, 0.950 .

Distribution of 121 MRNDs according to intent to surgery and related $p N+$ frequencies. Among the 121 NDs considered, $61 / 121(50 \%)$, presenting at least a clinical node involvement $(\mathrm{cN}+)$, were therapeutic NDs; 19/121 (16\%), presenting a cT3/cT4 and cN0 were elective NDs; the remaining 41/121 (34\%), presenting primary tumours small in size (cT1/cT2) and no evident clinical node metastases (cN0) but positive to sentinel lymph node metastases (SLN+) during the intraoperative assessment of sentinel lymph node, were NDs performed to establish the presence of other node metastases in addition to SLN+ (Table II). The distribution of $56 \mathrm{pN}+$ according to clinical-instrumental indications and, conversely, the $\mathrm{pN}+$ frequency in each indication are reported in Table II.

$p N+$ pattern. After excluding $19 \mathrm{pN}+$ cases due to lack of data useful to classify the $\mathrm{pN}+$ histopathological pattern, the remaining $37 \mathrm{pN}+$ NDs were distinguished into 17/37 with typical (46\%) and 20/37 with atypical (54\%) node metastases according to Woolgar's classification (21). The atypical pattern was largely represented by skip metastases [10/20 (50\%)], followed by the frequency of bilateral metastases [5/20 (25\%)], micrometastases [2/20 (10\%)], involvement of other lymph nodes alone [2/20 (10\%)], and 1/20 (5\%) showing both micrometastases and other lymph nodes co-interested (Fig. 3A). According to primary tumour site, skip metastases indiscriminately involved any site of the oral cavity, except cheek and fornix, which, on the contrary, preferentially showed micrometastases; bilateral metastases were frequently detected in OSCCs affecting tongue as a single site or in association with floor of the mouth or trigone; 'other different lymph nodes' were involved in floor of the mouth tumours (Fig. 3B and C).

Among $56 \mathrm{pN}+$ cases, $15 / 56 \mathrm{pN}+(26.8 \%)$ involved levels I-III, 11/56 pN+ (19.6\%) involved levels IV-V, and 30/56 $(53.6 \%) \mathrm{pN}+$ were censored due to level not reported.

ND anatomical levels I-III were typically involved by node metastases while levels IV-V showed $\mathrm{pN}+$ only for primary tumours of trigone, floor of the mouth plus tongue, tongue alone and multiple sites (data not shown).

Number of lymph nodes removed. A total of 3,390 lymph nodes were harvested in $121 \mathrm{NDs}$, mean $18.5 \pm 22.7$ per ND, range 1-110. One hundred and sixty-two lymph nodes were $\mathrm{pN}+$ in $56 \mathrm{NDs}$, mean $2.9 \pm 0.03$, range 1-10. 
Table II. pN+ distribution and frequency according to histological grading, AJCC staging and MRND intent.

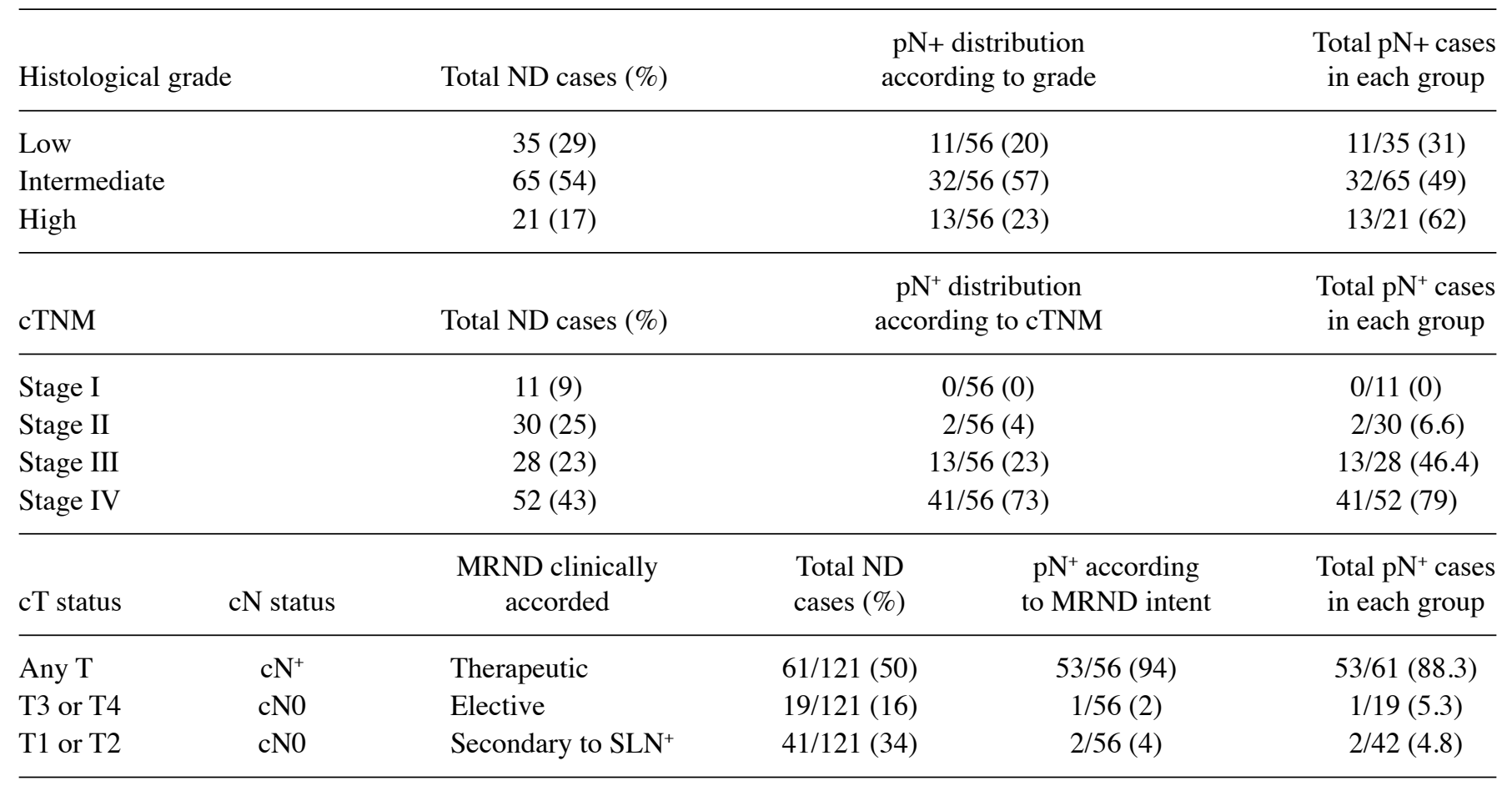

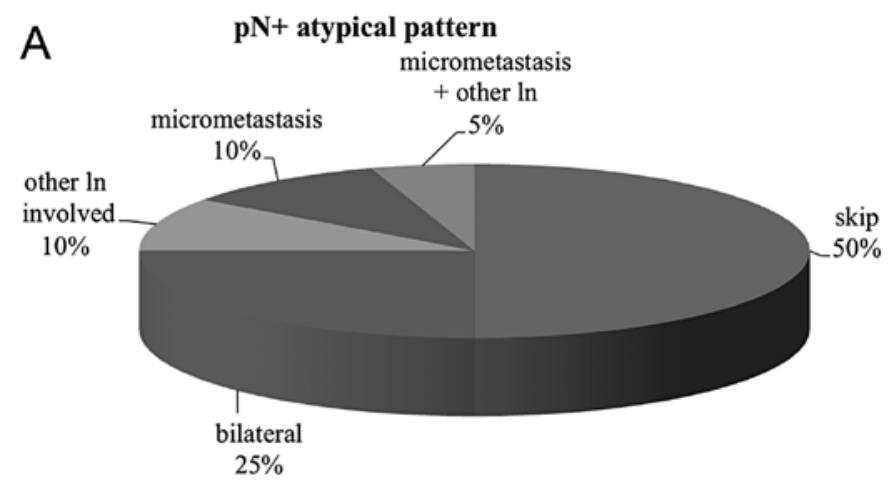

B Pattern of atypical metastases according to site

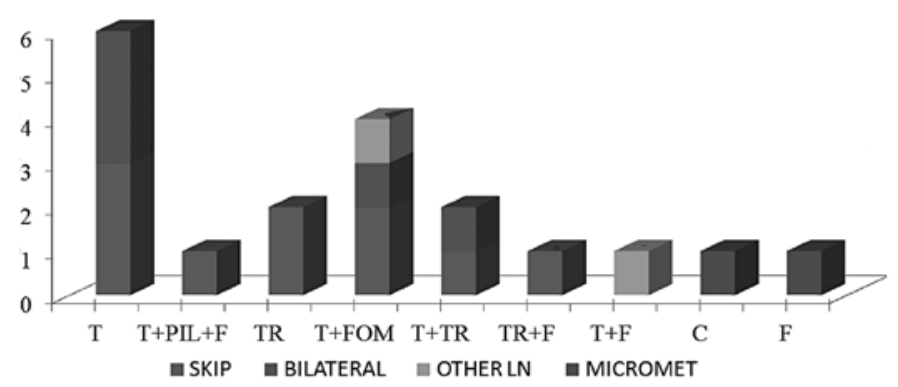

C

\begin{tabular}{ccccc}
\hline Site & Skip & Bilateral & Other Ln & Micromet \\
\hline T & 3 & 3 & 0 & 0 \\
T+PIL+F & 1 & 0 & 0 & 0 \\
TR & 2 & 0 & 0 & 0 \\
T+FOM & 2 & 1 & 1 & 0 \\
T+TR & 1 & 1 & 0 & 0 \\
TR+F & 1 & 0 & 0 & 0 \\
T+F & 0 & 0 & 1 & 0 \\
C & 0 & 0 & 0 & 1 \\
F & 0 & 0 & 0 & 1
\end{tabular}

Figure 3. Atypical metastatic pattern frequencies (A) and their distributions (B and C) according to primary tumour site. (C) Distribution of the 19 pN+ atypical metastases according to pattern and primary tumour site. The case showing both micrometastases and other lymph nodes co-interested has not been included. Ln, lymph node; T, tongue; PIL, pillars; F, fornix; FOM, floor of the mouth; TR, trigone; C, cheek.

Recurrence. Recurrence was observed in 8 cases, whose clinical, histological and lymph node features are shown in Table III.

Survival curves. Overall survival statistical significance and percentages of subjects alive at 1,2 and 5 years from the diagnosis are reported in Table IV.
Overall survival was statistically different according to early AJCC vs. late AJCC stages $(\mathrm{P}=0.0004)$ with a 5-year survival of 78.34 and $46.16 \%$, respectively, and according to absence/presence of nodal metastases ( $\mathrm{pN} 0$ vs. $\mathrm{pN}+, \mathrm{P}=0.0003$ ) with a 1-5 year overall survival ranging between $97-74 \%$ in pN0 cases and between $69-54 \%$ in $\mathrm{pN}+$ cases. 
Table III. Clinical, histological and lymph node features in the 8 cases with recurrence.

\begin{tabular}{lcllccc}
\hline Gender & Age (years) & \multicolumn{1}{c}{ T site } & Histological grade & AJCC stage & pN status & Outcome \\
\hline Female & 80 & FOM & Low & II & pN0 & Alive \\
Female & 83 & FOM & Intermediate & III & pN+ & Alive \\
Female & 48 & Tongue & Intermediate & IV & pN+ & Alive \\
Female & 70 & Tongue & Low & I & pN0 & Deceased \\
Male & 64 & Tongue & Intermediate & II & pN0 & Deceased \\
Male & 57 & FOM, Tongue & Intermediate & IV & pN+ & Alive \\
Male & 70 & Fornix & Intermediate & III & pN+ & Deceased \\
Male & 76 & Trigone & Intermediate & IV & pN0 & Deceased \\
\hline
\end{tabular}

Overall survival according to histological grade and typical vs. atypical lymph nodal metastatic pattern did not reach statistical significance when independently considered or when together. When comparing $\mathrm{pN} 0$ subjects, $\mathrm{pN}+$ with macrometastases or ECS and pN+ with micrometastases, patients reporting $\mathrm{pN}+$ with micrometastases showed an intermediate probability of survival after 5 years and these data were statistically significant $(\mathrm{P}=0.004)$. With regard to the number of lymph nodes harvested, statistically significant different survivals $(\mathrm{P}=0.005)$ were found in cases with $>30$ lymph nodes harvested, whose 5-year survival was $>80 \%$ compared to $46.93 \%$ in cases with $<20$ lymph nodes and $66.67 \%$ in the $20-30$ lymph node group, independently of the $\mathrm{pN}$ status.

With regard to intent to surgery, overall survival among subjects who had undergone elective, therapeutic and after SLN+ NDs were always statistically significant with the worst prognosis in patients who had undergone therapeutic ND and the most positive one in patients who had undergone ND after SLN+. pN status did not appear to be responsible for differences in survival since patients with pN0/therapeutic MRND showed a $15 \%$ 5-year survival compared to $\mathrm{pN}+/$ therapeutic MRND patients with a $45.72 \%$ 5-year survival.

\section{Discussion}

In the present study, a descriptive and statistical retrospective study on 121 OSCCs who had undergone neck dissection (ND) was conducted, focusing on metastatic pattern (typical vs. atypical), number of lymph nodes harvested and node metastasis features in terms of size, anatomical extent and surgical decision orienting the ND (elective, therapeutic and after SLN+).

Correlation among histological malignancy grading in OSCC and different clinical parameters such as clinical staging, recurrence and prognosis have been published in different studies and a close relationship between the degree of histological differentiation and the incidence of lymph node metastasis (LNM) has been reported by several investigators in an attempt to identify a better prognosis. Our results are partly in accordance with previous literature (23).

Descriptive data showed, as expected, that the more the primitive tumour was towards undifferentiation and AJCC advanced staging, the more frequent the nodal metastases. Despite these findings, statistically significant differences in the overall survival were observed only in relation to AJCC staging and not according to histological grading.

Since $88.3 \%$ of NDs performed for therapeutic intent presented at least one $\mathrm{pN}+$, the therapeutic value was confirmed. On the contrary, the low $\mathrm{pN}+$ frequencies in elective NDs $(5.3 \%)$ and in NDs secondary to SLN+ $(4.8 \%)$ revealed an overtreatment in the remaining 94.7 and $95.2 \%$ of cases, respectively. $\mathrm{pN}+$ was found in 2 out of 42 NDs performed after SLN+ $(0 / 11,0 \%, \mathrm{pN}+$ in cT1-cN0M0 NDs and 2/31,6.45\%, in cT2-cN0M0 NDs), thus confuting previous literature supporting its role as a diagnostic marker for other nodal metastases $(24,25)$ and bringing the predictive role of SLN positivity into discussion, leading us to conclude that a ND secondary to SLN+ is an overtreatment in $100 \%$ of cT1-cN0M0 NDs and in $93.55 \%$ of cT2-cN0M0 NDs. We suggest conducting further biomolecular studies focusing on molecular markers able to predict occult metastatic disease in SLN biopsies, thereby improving the quality of the treatments (26).

The significant association between clinical staging and histopathological report, confirmed by high accuracy (90.9\%) revealed the quality of the pre-surgical clinical and instrumental staging of the tumours.

With regard to the anatomical lymph nodal levels involved, results showing involvement of levels IV-V only for primary tumours of trigone, floor of the mouth plus tongue, tongue alone and multiple sites, express the major morbidity of the OSCCs of these primary sites, requesting an ND extended until level V and lead us to re-consider the extended ND in the cases of OSCC affecting different oral sites such as cheek, which never showed levels of IV-V involvement in the present study. Shah (27) provided evidence that the pattern of neck metastasis of carcinomas from upper aerodigestive tract is predictable based on the location of the primary lesion. Moreover, it has been shown that in patients with oral carcinoma and clinical evidence of neck disease, the rate of pathologic involvement of level $\mathrm{V}$ nodes was only $4 \%$. In the present study, the percentage of pathologic involvement of level IV-V nodes was $19.6 \%$, higher when compared with the $4 \%$ value reported by Shah (27).

Recurrences, observed in 8 cases, mainly occurred in floor of the mouth and tongue OSCCs, at intermediate grade of differentiation and independently from the $\mathrm{pN}$ status.

The survival probability was in accordance with the number of lymph nodes harvested, as previously demonstrated by Amar et al (6), who found the larger number of lymph nodes 
Table IV. One-, 2- and 5-year overall survival and the statistical significance.

\begin{tabular}{|c|c|c|c|c|}
\hline & 1 -year $(\%)$ & 2-year (\%) & 5-year $(\%)$ & $\mathrm{P}<0.05$ \\
\hline Stage I-II & 90.00 & 87.50 & 78.34 & Yes \\
\hline Stage III-IV & 76.16 & 50.78 & 46.16 & \\
\hline Low grade & 83.24 & 68.65 & 68.65 & No \\
\hline Intermediate-high grade & 81.11 & 64.95 & 51.45 & \\
\hline pNo & 93.54 & 94.54 & 69.11 & Yes \\
\hline $\mathrm{pN}+$ & 66.03 & 45.41 & 45.41 & \\
\hline $\mathrm{pN}+/$ Stage I-II ${ }^{\mathrm{a}}$ & 0 & 0 & 0 & Yes \\
\hline $\mathrm{pN}+/$ Stage III-IV & 67.46 & 46.20 & 46.20 & \\
\hline pN+/Low grade & 50.00 & 41.66 & 41.66 & No \\
\hline $\mathrm{pN}+/$ Intermediate-high grade & 69.35 & 44.56 & 44.56 & \\
\hline Typical pN+ & 50.89 & 44.53 & 44.53 & No \\
\hline Atypical pN+ & 72.22 & 41.27 & 41.27 & \\
\hline Typical pN+/Low grade & 50.00 & 50.00 & 50.00 & No \\
\hline Typical pN+/Intermediate-high grade & 51.56 & 51.56 & 51.56 & \\
\hline Atypical pN+/Low grade & 78.57 & 34.37 & 34.37 & \\
\hline Atypical $\mathrm{pN}+/$ Intermediate-high grade & 50.00 & 25.00 & 25.00 & \\
\hline pN+ with ECS/macrometastases & 80.67 & 28.68 & 28.68 & Yes \\
\hline $\mathrm{pN}+$ with micrometastases & 80.00 & 40.00 & 40.00 & \\
\hline pNo & 93.54 & 81.50 & 69.10 & \\
\hline $\mathrm{LN}<20$ & 72.38 & 52.36 & 46.93 & Yes \\
\hline LN 20-30 & 88.80 & 66.67 & 66.67 & \\
\hline $\mathrm{LN}>30$ & 96.29 & 87.59 & 80.60 & \\
\hline pN0/Elective & 87.50 & 79.54 & 62.64 & Yes \\
\hline pN0/Therapeutic & 87.50 & 30.00 & 15.00 & \\
\hline pN0/SLN+ & 100.00 & 94.51 & 84.63 & \\
\hline $\mathrm{pN}+/$ Elective & 100.00 & 100.00 & 100.00 & \\
\hline $\mathrm{pN}+/$ Therapeutic & 68.10 & 45.71 & 45.71 & \\
\hline $\mathrm{pN}+/ \mathrm{SLN}+{ }^{\mathrm{b}}$ & 0 & 0 & 0 & \\
\hline SLN+ & 95.00 & 89.79 & 80.40 & Yes \\
\hline $\mathrm{E}$ & 88.23 & 80.88 & 65.36 & \\
\hline $\mathrm{T}$ & 70.745 & 42.86 & 39.56 & \\
\hline $\mathrm{pN}+/ \mathrm{SLN}+$ & 100.00 & 95.52 & 84.63 & Yes \\
\hline $\mathrm{pN}+/ \mathrm{E}$ & 100.00 & 100.00 & 100.00 & \\
\hline $\mathrm{pN}+/ \mathrm{T}$ & 68.11 & 45.72 & 45.72 & \\
\hline pN0/SLN+ & 100.00 & 94.52 & 84.63 & Yes \\
\hline $\mathrm{pN} 0 / \mathrm{E}$ & 87.50 & 71.59 & 62.64 & \\
\hline $\mathrm{pN} 0 / \mathrm{T}$ & 87.50 & 30.00 & 15.00 & \\
\hline
\end{tabular}

aThere were $2 \mathrm{pN}^{+} /$Stage I-II subjects. ${ }^{b}$ The 2 patients $\mathrm{pN}^{+} / \mathrm{SLN}^{+}$died after 9 months.

dissected in the ND related to the group of better prognoses among pN0 cases, while we found this result independently from the $\mathrm{pN}$ status.

We also confirmed the statistically significant different survival probability of patients with micrometastases (5-year, $40 \%$ ), intermediate among pN0 (5-year, 69.10\%) and pN+ with macrometastases patients (5-year, $28.68 \%$ ), as previously reported by Broglie et al (20) and Han et al (28).
Statistically significant difference in the overall survival was also found according to clinical intent to surgery independently from the $\mathrm{pN} 0$, with a better prognosis in patients who had undergone elective ND or after $\mathrm{SLN}^{+}$when compared with the ones who had undergone therapeutic ND, independently from $\mathrm{pN}$ status.

In conclusion, the surgical management of regional metastatic neck disease in patients with oral and oropharyngeal 
cancer remains a topic of debate and controversy. For several years classical comprehensive ND has been the mainstay of treatment. Selective and super-selective neck treatments were recently introduced and widely applied in order to reduce morbidity and mortality related to extensive ND, thus improving the postoperative quality of life after preservation of level $\mathrm{V}$ and the surrounding anatomical structures.

With regard to atypical metastases according to Woolgar's definitions, no statistically significant differences were found related to the overall survival of this group vs. typical metastatic pattern. However, we noted different atypical features related to different primary tumour sites. Our data suggest that enhancing our knowledge of these types of atypical patterns site-related, and further evaluations such as novel imaging techniques (29) and molecular analyses (30) may help us to understand if any behaviour and biomolecular differences exist among OSCCs according to site.

\section{References}

1. Saman DM: A review of the epidemiology of oral and pharyngeal carcinoma: update. Head Neck Oncol 4: 1-7, 2012.

2. Greene FL, Page DL, Fleming ID, Fritz AG, Balch CM, Haller DG and Morrow M (eds). AJCC Cancer Staging Handbook. From the AJCC Cancer Staging Manual. 6th edition. Springer-Verlag, 2002.

3. Edge SB and Compton CC: The American Joint Committee on Cancer: the 7th edition of the AJCC cancer staging manual and the future of TNM. Ann Surg Oncol 17: 1471-1474, 2010.

4. Wenzel S, Sagowski C, Kehrl W and Metternich FU: The prognostic impact of metastatic pattern of lymph nodes in patients with oral and oropharyngeal squamous cell carcinomas. Eur Arch Otorhinolaryngol 261: 270-275, 2004.

5. Kowalski LP and Medina JE: Nodal metastases: predictive factors. Otolaryngol Clin North Am 31: 621-637, 1998.

6. Amar A, Chedid HM, Rapoport A, Cernea CR, Dedivitis RA, Curioni OA and Brandão LG: Prognostic significance of the number of lymph nodes in elective neck dissection for tongue and mouth floor cancers. Braz J Otorhinolaryngol 78: 22-26, 2012.

7. Compton CC and Byrd DR: Lip and oral cavity. In: 2nd AJCC Cancer Staging Atlas. Compton CC, Byrd DR, Garcia-Aguilar J, Kurtzman SH, Olawaiye A and Washington MK (eds). Springer, New York, pp41-53, 2012.

8. Ferlito A, Robbins KT, Shah JP, Medina JE, Silver CE, Al-Tamimi S, Fagan JJ, Paleri V, Takes RP, Bradford CR, Devaney KO, Stoeckli SJ, Weber RS, Bradley PJ, Suárez C, Leemans CR, Coskun HH, Pitman KT, Shaha AR, de Bree R, Hartl DM, Haigentz M Jr, Rodrigo JP, Hamoir M, Khafif A, Langendijk JA, Owen RP, Sanabria A, Strojan P, Vander Poorten V, Werner JA, Bień S, Woolgar JA, Zbären P, Betka J, Folz BJ, Genden EM, Talmi YP, Strome M, González Botas JH, Olofsson J, Kowalski LP, Holmes JD, Hisa Y and Rinaldo A: Proposal for a rational classification of neck dissections. Head Neck 33: 445-450, 2011.

9. Byers RM, Wolf PF and Ballantyne AJ: Rationale for elective modified neck dissection. Head Neck Surg 10: 160-167, 1988.

10. Pagedar NA and Gilbert RW: Selective neck dissection: a review of the evidence. Oral Oncol 45: 416-420, 2009.

11. Ferlito A, Robbins KT, Silver CE, Hasegawa Y and Rinaldo A: Classification of neck dissections: an evolving system. Auris Nasus Larynx 36: 127-134, 2009.

12. Bernier J, Domenge C, Ozsahin M Matuszewska K, Lefèbvre JL, Greiner RH, Giralt J, Maingon P, Rolland F, Bolla M, Cognetti F, Bourhis J, Kirkpatrick A and van Glabbeke M: Postoperative irradiation with or without concomitant chemotherapy for locally advanced head and neck cancer. N Engl J Med 350: 1945-1952, 2004.
13. Cooper JS, Pajak TF, Forastiere AA, Jacobs J, Campbell BH, Saxman SB, Kish JA, Kim HE, Cmelak AJ, Rotman M, Machtay M, Ensley JF, Chao KS, Schultz CJ, Lee N and Fu KK: Postoperative concurrent radiotherapy and chemotherapy for high-risk squamous-cell carcinoma of the head and neck. N Engl J Med 350: 1937-1944, 2004.

14. van den Brekel MW, van der Waal I, Meijer CJ, Freeman JL, Castelijns JA and Snow GB: The incidence of micrometastases in neck dissection specimens obtained from elective neck dissections. Laryngoscope 106: 987-991, 1996.

15. Murer K, Huber GF, Haile SR and Stoeckli SJ: Comparison of morbidity between sentinel node biopsy and elective neck dissection for treatment of the $\mathrm{n} 0$ neck in patients with oral squamous cell carcinoma. Head Neck 33: 1260-1264, 2011.

16. Schiefke F, Akdemir M, Weber A, Akdemir D, Singer S and Frerich B: Function, postoperative morbidity, and quality of life after cervical sentinel node biopsy and after selective neck dissection. Head Neck 31: 503-512, 2009.

17. Broglie MA, Haile SR and Stoeckli SJ: Long-term experience in sentinel node biopsy for early oral and oropharyngeal squamous cell carcinoma. Ann Surg Oncol 18: 2732-2738, 2011.

18. Teymoortash A, Hoch S, Eivazi B and Werner JA: Postoperative morbidity after different types of selective neck dissection. Laryngoscope 120: 924-929, 2010.

19. Robbins KT, Shaha AR, Medina JE, Califano JA, Wolf GT, Ferlito A, Som PM and Day TA; Committee for Neck Dissection Classification, American Head and Neck Society: Consensus statement on the classification and terminology of neck dissection. Arch Otolaryngol Head Neck Surg 134: 536-538, 2008.

20. Broglie MA, Haerle SK, Huber GF, Haile SR and Stoeckli SJ: Occult metastases detected by sentinel node biopsy in patients with early oral and oropharyngeal squamous cell carcinomas: impact on survival. Head Neck 35: 660-666, 2013.

21. Woolgar JA: The topography of cervical lymph node metastases revisited: the histological findings in 526 sides of neck dissection from 439 previously untreated patients. Int J Oral Maxillofac Surg 36: 219-225, 2007.

22. Woolgar JA, Scott J, Vaughan ED, Brown JS, West CR and Rogers S: Survival, metastasis and recurrence of oral cancer in relation to pathological features. Ann R Coll Surg Engl 77: 325-331, 1995.

23. Shah JP: Surgical approaches to the oral cavity primary and neck. Int J Radiat Oncol Biol Phys 69: S15-S18, 2007.

24. Stoeckli SJ, Alkureishi LW and Ross GL: Sentinel node biopsy for early oral and oropharyngeal squamous cell carcinoma. Eur Arch Otorhinolaryngol 266: 787-793, 2009.

25. Ross GL, Soutar DS, MacDonald DG, Shoaib T, Camilleri IG and Robertson AG: Improved staging of cervical metastases in clinically node-negative patients with head and neck squamous cell carcinoma. Ann Surg Oncol 11: 213-218, 2004.

26. Huber GF, Züllig L, Soltermann A Roessle M, Graf N, Haerle SK, Studer G, Jochum W, Moch H and Stoeckli SJ: Down regulation of E-Cadherin (ECAD) - a predictor for occult metastatic disease in sentinel node biopsy of early squamous cell carcinomas of the oral cavity and oropharynx. BMC Cancer 11: $217,2011$.

27. Shah JP: Pattern of cervical node metastasis from squamous carcinomas of the upper aerodigestive tract. Am J Surg 160: 405-409, 1990

28. Han MW, Cho KJ, Roh JL, Choi SH, Nam SY and Kim SY: Patterns of lymph node metastasis and their influence on outcomes in patients with submandibular gland carcinoma. J Surg Oncol 106: 475-480, 2012.

29. Contaldo M, Agozzino M, Moscarella E, Esposito S, Serpico R and Ardigò $\mathrm{M}$ : In vivo characterization of healthy oral mucosa by reflectance confocal microscopy: a translational research for optical biopsy. Ultrastruct Pathol 37: 151-158, 2013.

30. Di Domenico M, Pierantoni GM, Feola A Esposito F, Laino L, DE Rosa A, Rullo R, Mazzotta M, Martano M, Sanguedolce F, Perillo L, D'Angelo L, Papagerakis S, Tortorella S, Bufo P, Lo Muzio L, Pannone G and Santoro A: Prognostic significance of $\mathrm{N}$-Cadherin expression in oral squamous cell carcinoma. Anticancer Res 31: 4211-4218, 2011. 